
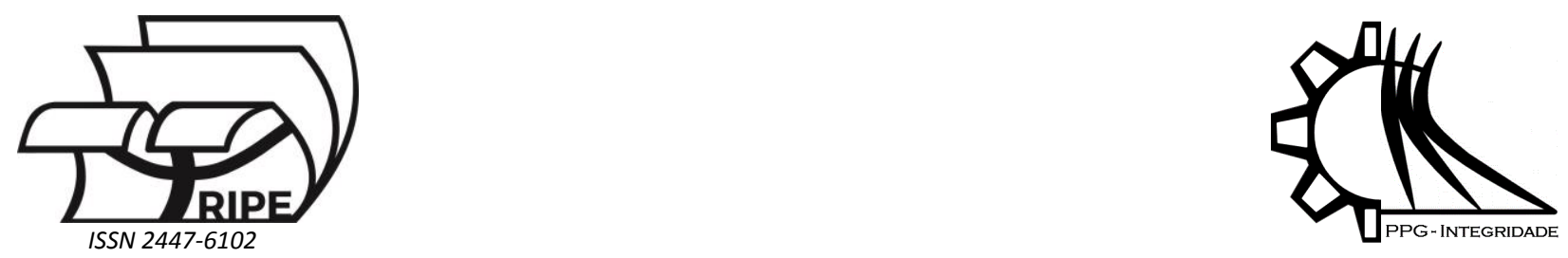

Article

\title{
Determinação da probabilidade de falha em um edifício submetido a uma excitação sísmica
}

\author{
Chiesa, D. D. ${ }^{1, *}$ and Miguel, L. F. F. ${ }^{2}$ \\ 1 Departamento de Engenharia Mecânica, Universidade Federal do Rio Grande do Sul; daniela.chiesa@ufrgs.br \\ 2 Departamento de Engenharia Mecânica, Universidade Federal do Rio Grande do Sul;,letffm@ufrgs.br \\ * Correspondente: daniela.chiesa@ufrgs.br
}

Received: 14/12/2018; Accepted: 05/01/2019; Published: 15/01/2019

Resumo: Este artigo apresenta uma metodologia para a determinação da probabilidade de falha em edifícios submetidos a uma excitação sísmica. Incertezas nos parâmetros do movimento do solo e da estrutura são consideradas nesse procedimento. A excitação sísmica é modelada como um processo estocástico estacionário unidimensional, utilizando a função densidade espectral de potência de Kanai-Tajimi, e são atribuídas aleatoriedades na frequência, amortecimento e pico de aceleração do solo. A estrutura analisada corresponde a um edificio, com 10 pavimentos, no qual a obtenção da resposta dinâmica é calculada levando-se em conta as aleatoriedades em sua massa, rigidez e razão de amortecimento. Para ilustrar a proposta, são realizadas 3.000 simulações computacionais, no programa MATLAB, considerando três tipos de solos, em uma região que poderia estar situada na zona sísmica 3 do Peru. Os valores máximos médios, em módulo, para o deslocamento e a aceleração em cada andar do edifício, o deslocamento relativo entre os andares e a probabilidade de falha são determinados. Considera-se como estado limite para dano estrutural um valor máximo para o deslocamento relativo entre os andares. Os resultados mostram que altas probabilidades de falhas são observadas em solos com frequências situadas entre as frequências naturais dos dois primeiros modos de vibrações da estrutura.

Palavras-chave: Probabilidade de falha. Excitação sísmica. Kanai-Tajimi. Parâmetros aleatórios.

\section{Determination of the failure probability in a building subjected to a seismic excitation}

Abstract: This paper presents a methodology for the determination of the failure probability of buildings subjected to a seismic excitation. Uncertainties in the parameters of ground and of structure are considered. The seismic excitation is defined as a one-dimensional stochastic process, which is simulated by passing a Gaussian white noise process through a Kanai-Tajimi filter, in which the frequency, damping and peak ground acceleration are random parameters. A 10-storey building is analyzed, in which the dynamic response is calculated taking into account the randomness in its mass, stiffness and damping ratio. For illustration purposes, 3,000 computational simulations, in MATLAB software, are carried, considering three types of soils in a region located in Peru. The mean maximum values, in modulus, of the displacements and accelerations, the inter-storey drift and the failure probability of the building are calculate. A maximum value for the interstorey drift are considered as a limit state for damage. The results show that high failure probabilities are obtained in soils with frequencies located between the natural frequencies of the first two vibration modes of the structure.

Keywords: Failure probability. Seismic excitation. Kanai-Tajimi. Random parameters. 
Os terremotos representam uma grande ameaça à vida e aos bens materiais dos seres humanos, devido a seus efeitos secundários tais como tsunamis, deslizamentos de terras ou danos provocados às construções. Para lidar com as perdas materiais e mortes provocadas por um abalo sísmico, há anos estudiosos tentam melhorar modelos relacionados à previsão de terremotos e modelos que descrevam da melhor forma possível o fenômeno, entretanto, devido a sua aleatoriedade, atualmente, não é possível prever de forma eficaz o local ou o momento exato que abalos sísmicos ocorrerão ou, ainda, estimar com precisão a sua amplitude ou conteúdo de frequência. Nesse contexto, uma alternativa que se torna factível é a adoção de medidas para reduzir o seu potencial destrutivo. Dentre essas medidas estão à elaboração de projetos sismo-resistentes.

Durante um terremoto a base de uma estrutura é submetida à aceleração do solo e esse movimento acaba provocando na mesma, esforços e deformações. Consequentemente, para a elaboração de um projeto sismo-resistente é necessário construir um modelo probabilístico que leve em conta dados como as características do solo da região a qual está localizada a estrutura. Porém, as respostas estruturais podem apresentar valores que estão distantes dos reais devido aos dados considerados na elaboração do modelo. Aleatoriedades nos parâmetros do movimento do solo e da estrutura parecem reproduzir de forma mais realista todo esse contexto, uma vez que as variáveis envolvidas no estudo não podem ser medidas com precisão e por menor que seja sempre existirão variações em seus valores.

Partindo de todos esses pressupostos, este estudo tem como objetivo, primeiramente, modelar um evento sísmico considerando incertezas nos parâmetros do movimento do solo, isto é, na frequência, amortecimento e pico de aceleração. Com essa finalidade, as excitações sísmicas são modeladas como processos estocásticos estacionários unidimensionais utilizando a função densidade espectral de potência de Kanai-Tajimi, função esta, que tem sido muito bem aceita pela comunidade científica e, em virtude disso, adotada em vários trabalhos (por exemplo: Miguel, Miguel e Lopez (2016b), Mohebbi et al. (2013), Seya, Talbott e Hwang (1993) e Singh e Moreschi (2001)).

Após a geração do sinal sísmico, simulações numéricas são realizadas, no programa MATLAB, visando à obtenção da resposta dinâmica de um edifício de 10 andares. Nessas simulações são adotados três tipos de solos e também são inseridas aleatoriedades nos parâmetros estruturais, ou seja, na massa, rigidez e razão de amortecimento. Especificamente, os valores máximos médios, em módulo, para o deslocamento e a aceleração em cada andar do edifício, o deslocamento relativo entre os andares e a probabilidade de falha são determinados. Na determinação da probabilidade de falha considera-se como estado limite para dano estrutural um valor máximo para o deslocamento relativo entre os andares.

\section{Metodologia}

\subsection{Equação do Movimento}

A equação de movimento de um sistema, com $n$ graus de liberdade (GDL), sujeito a uma excitação aleatória em sua base é dada por (Miguel, Miguel e Lopez 2016b)):

$$
\boldsymbol{M} \ddot{\mathbf{z}}(t)+\boldsymbol{C} \dot{\boldsymbol{z}}(t)+\boldsymbol{K} \boldsymbol{z}(t)=-\boldsymbol{M B} \ddot{\boldsymbol{y}}(t),
$$

onde:

$\boldsymbol{M}, \boldsymbol{C}, \boldsymbol{K}=$ são as matrizes, de ordem $n$, de massa, amortecimento e rigidez, respectivamente;

$\ddot{\mathbf{z}}(t), \dot{\boldsymbol{z}}(t), \quad \boldsymbol{z}(t)=$ são os vetores de aceleração, velocidade e deslocamento relativos do sistema, respectivamente;

$\boldsymbol{B}$ = é a matriz que contém os cossenos diretores dos ângulos formados entre o movimento da base e as direções dos GDL;

$\ddot{\boldsymbol{y}}(t)$ = é o vetor de aceleração na base.

\subsection{Geração da Excitação Sísmica}

O vetor $\ddot{\boldsymbol{y}}(t)$ é definido como um carregamento sísmico unidimensional que é simulado passando um processo ruído branco gaussiano através do filtro Kanai-Tajimi (Kanai 1961; Tajimi 1960), com função densidade espectral de potência $S(\omega)$ dada por: 


$$
S(\omega)=S_{0} \frac{\omega_{s}^{4}+4 \omega_{s}^{2} \xi_{s}^{2} \omega^{2}}{\left(\omega^{2}-\omega_{s}^{2}\right)^{2}+4 \omega_{s}^{2} \xi_{s}^{2} \omega^{2}}
$$

onde:

$S_{0}=$ densidade espectral constante;

$\omega$ = frequência de entrada;

$\omega_{s}=$ frequência do solo;

$\xi_{s}=$ amortecimento do solo.

A Eq. (2) está no domínio da frequência. Para gerar um sinal de aceleração do solo no domínio do tempo é utilizado o método proposto por Shinozuka e Jan (1972). Após a obtenção do sinal no domínio do tempo, o mesmo é normalizado de forma que o valor de pico de aceleração do solo (PGA) esteja de acordo com as características do solo da região em estudo.

\subsection{Dados Estruturais}

A estrutura em estudo corresponde a um edifício de 10 andares $(n=10)$, cuja altura de cada andar $h_{i}$ é 3 metros e no qual o material predominante é o aço. Os valores correspondentes a massa e rigidez, em cada andar, são dados, respectivamente, por:

$$
m_{i}=360.000 \mathrm{Kg}, \quad k_{i}=650 \mathrm{MN} / \mathrm{m}, \quad i=1,2.3, \ldots n
$$

Adota-se uma razão de amortecimento $\xi$, referente aos dois primeiros modos de vibrações da estrutura, de 0,01 . Os valores das frequências naturais do edifício são: $6,35 \mathrm{rad} / \mathrm{s}, 18,91 \mathrm{rad} / \mathrm{s}, 31,05 \mathrm{rad} / \mathrm{s}, 42,49 \mathrm{rad} / \mathrm{s}, 52,99 \mathrm{rad} / \mathrm{s}$, $62,30 \mathrm{rad} / \mathrm{s}, 70,22 \mathrm{rad} / \mathrm{s}, 76,57 \mathrm{rad} / \mathrm{s}, 81,21 \mathrm{rad} / \mathrm{s}$ e 84,03 rad/s.

\subsection{Incertezas nos Parâmetros do Sismo e da Estrutura}

Parâmetros do movimento do solo e parâmetros estruturais, como a resistência dos materiais, sempre exibem alguma variação estatística sendo assim, uma abordagem probabilística torna-se apropriada para avaliação da resposta estrutural, porque pode incorporar esses tipos de incertezas na construção de um modelo (Seya, Talbott e Hwang 1993). O modelo probabilístico sísmico é desenvolvido com vários parâmetros aleatórios no movimento do solo e na estrutura analisada. As incertezas no movimento do solo são inseridas nos parâmetros $\omega_{s}$, $\xi_{s}$ e PGA utilizadas na geração do sinal sísmico, consequentemente, cada vez que uma simulação é executada um histórico de tempo para a aceleração do solo é obtido. As incertezas na estrutura são introduzidas nos parâmetros $m_{i}, k_{i}$ e $\xi$, assim a cada simulação os valores referentes às frequências naturais da estrutura sofrem pequenas alterações.

Todos esses parâmetros aleatórios, do movimento do solo e da estrutura, representam as variáveis aleatórias do problema. Essas variáveis aleatórias são modeladas como variáveis Gaussianas independentes com valores predeterminados de média e de coeficiente de variação $C V$, procedimento como este foi desenvolvido no trabalho de (Miguel, Miguel e Lopez 2016a). Na Tab. 1 são informados os $\mathrm{CV}_{\mathrm{s}}$ utilizados para a geração da sequência de números de cada variável aleatória.

\subsection{Estado Limite e Probabilidade de Falha}

$\mathrm{Na}$ elaboração de um projeto estrutural deve-se observar o fato de que a estrutura precisa ser projetada e, posteriormente, construída de forma a resistir aos efeitos das excitações sísmicas. Um estado limite indica um estado de comportamento estrutural indesejável. No caso de um abalo sísmico danos estruturais podem ser atribuídos aos deslocamentos excessivos entre os andares, portanto, uma boa medida de falhas estruturais pode ser determinada analisando os deslocamentos relativos máximos entre os andares da estrutura (Seya, Talbott e Hwang 1993). Nesse contexto, utiliza-se como estado limite os deslocamentos relativos $\Delta i(\Delta i=i-(i-1))$, de um andar $i$, definidos pelo Decreto Supremo no. 003-2016 (PERU 2016). A escolha desse decreto fundamenta-se no fato de que as excitações sísmicas foram elaboradas com o valor do PGA médio correspondente a regiões peruanas. Assim, os limites de deslocamentos relativos $\Delta i$, considerando-se um edifício cujo material predominante é o aço, são dados pela seguinte equação (PERU 2016): 
Tabela 1. Variáveis aleatórias e seus respectivos CVs.

\begin{tabular}{cc}
\hline Variáveis aleatórias & $\mathrm{CV}$ \\
\hline$\omega_{s}$ & 0,2 \\
$\xi_{s}$ & 0,2 \\
$\mathrm{PGA}$ & 0,1 \\
$m_{i}$ & 0,05 \\
$k_{i}$ & 0,05 \\
$\xi$ & 0,1 \\
\hline
\end{tabular}

\section{Resultados}

A rotina computacional, implementada no programa MATLAB, tem como objetivo calcular a probabilidade de falha e as médias dos valores máximos, em módulo, dos deslocamentos e acelerações em cada andar e o valor máximo médio dos deslocamentos relativos entre os andares, de um edifício, com $n$ GDL, sujeito a uma excitação sísmica na sua base.

As variáveis aleatórias, utilizadas no cálculo da função densidade espectral de Kanai-Tajimi, possuem valores predeterminados de média, que variam conforme o tipo de solo da região em estudo e CV (ver Tab. 1). Para solos do tipo mole $\omega_{s}$ é estimado no intervalo de $2,4 \pi$ a $3,5 \pi$, para solos do tipo rocha $\omega_{s}$ varia de $8 \pi$ a $10 \pi$ e para solos rígidos $\omega_{s}$ é determinado entre os valores de solos do tipo rocha e solos moles (Seya, Talbott e Hwang 1993). O valor do PGA médio adotado, corresponde ao valor de regiões localizadas na zona sísmica 3 do Peru. A Tab. 2 mostra o valor médio adotado para cada variável aleatória, em cada tipo de solo, e a duração do sismo (T).

Tabela 2. Valores médios das variáveis aleatórias conforme o tipo de solo.

\begin{tabular}{ccccc}
\hline Tipo de sololVariável & $\omega_{s}(\mathrm{rad} / \mathrm{s})$ & $\xi_{s}$ & $P G A\left(\mathrm{~g} \approx 9,81 \mathrm{~m} / \mathrm{s}^{2}\right)$ & $T(\mathrm{~s})$ \\
\hline Rocha & $8 \pi$ & 0,6 & $0,35 g$ & 15 \\
Rígido & $5 \pi$ & 0,6 & $0,35 g$ & 20 \\
Mole & $3 \pi$ & 0,85 & $0,35 \mathrm{~g}$ & 25 \\
\hline
\end{tabular}

Com o intuito de verificar a convergência dos resultados foram realizadas 1.000 simulações, considerando um valor nulo para o CV de cada variável aleatória e um solo do tipo rocha. As Figs. 1 e 2 mostram as curvas de convergência da variância e do valor máximo médio, em módulo, respectivamente, para o deslocamento referente ao 10 o andar. Observando as Figs. 1 e 2 é possível verificar que são necessárias, no mínimo, 500 simulações para estabilizar as curvas de convergências do deslocamento, o mesmo ocorre para as curvas de convergência referentes à aceleração e ao deslocamento relativo entre os andares. As curvas de convergência referentes aos outros andares da estrutura exibem um comportamento similar. A Fig. 3 ilustra a função densidade espectral de Kanai-Tajimi com os dados descritos na Tab. 2. Para a geração do sinal sísmico, no domínio do tempo, os seguintes dados são utilizados: frequência mínima $f_{\min }=0 \mathrm{~Hz}$, frequência máxima $f_{\text {max }}=25 \mathrm{~Hz}$, incremento de frequência $\Delta f=0,025 \mathrm{~Hz}$, tempo inicial $t_{0}=0 \mathrm{~s}$ e incremento de tempo $\Delta t=0,02 \mathrm{~s}$. Para a análise da resposta dinâmica da estrutura utiliza-se o método de Newmark. A Fig. 4 exemplifica um histórico de acelerações de um sinal sísmico. 


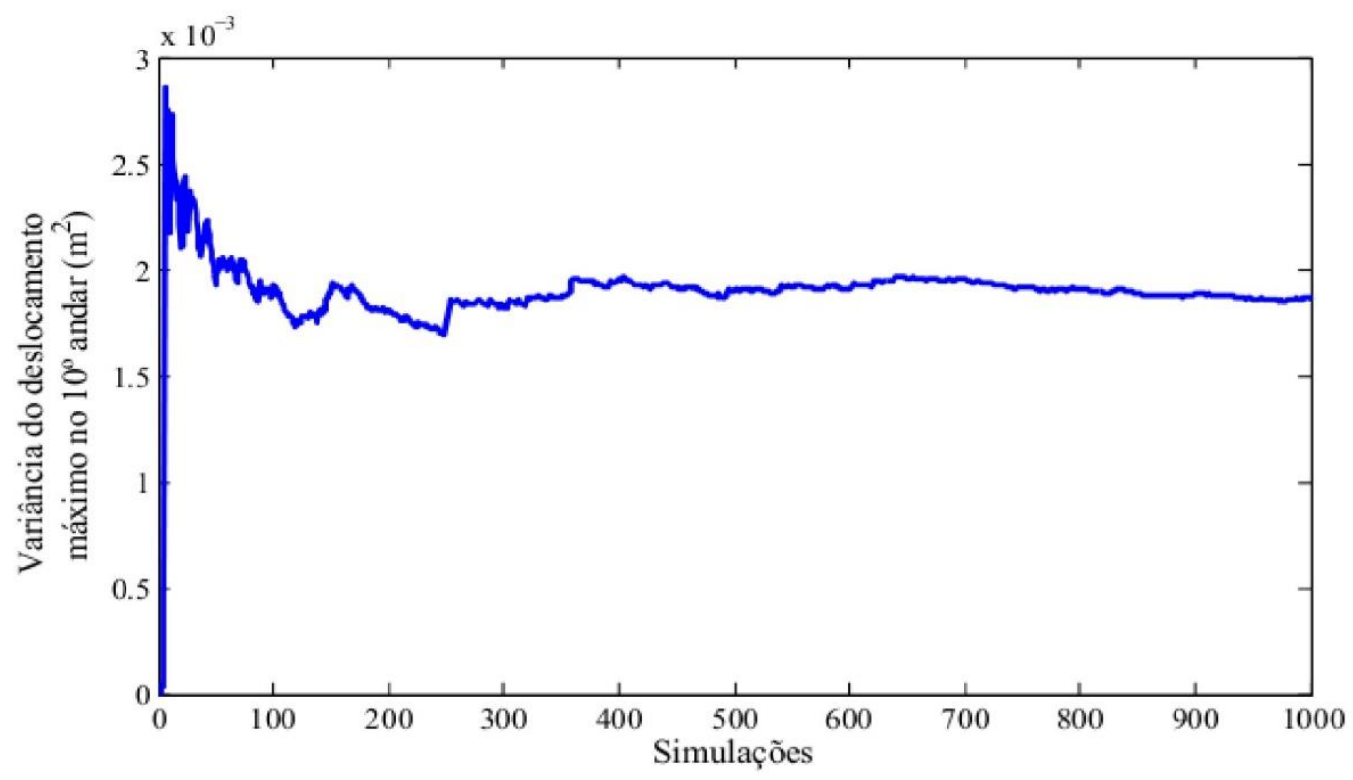

Figura 1: Variância do deslocamento.

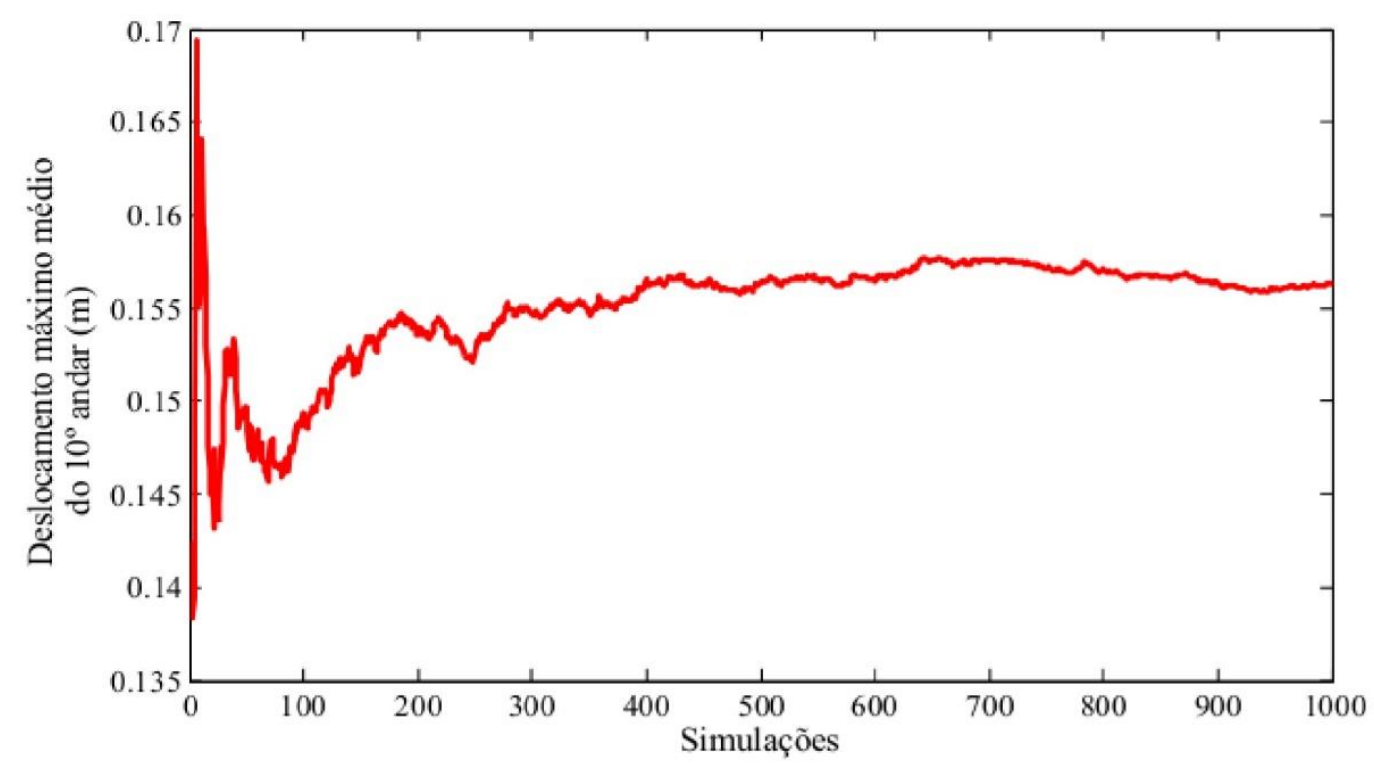

Figura 2: Deslocamento máximo médio.

Os resultados numéricos, apresentados na sequência, representam as soluções médias de 1.000 simulações numéricas para três tipos de solos, totalizando 3.000 simulações. As Tabs. 3, 4 e 5 mostram os resultados médios obtidos, referentes às simulações nos três tipos de solos. As primeiras colunas indicam os andares do edifício, as segundas colunas referem-se às médias dos deslocamentos máximos, em módulo, de cada andar $M\left(\max \left|z_{i}\right|\right)$, as terceiras colunas mostram os resultados dos valores médios das acelerações máximas, em módulo, de cada andar $M\left(\max \left|\ddot{z}_{i}\right|\right)$ e as últimas colunas apresentam as médias dos deslocamentos relativos máximos entre os andares $M\left(\max \left|\Delta_{i}\right|\right)$. A Tab. 6 ilustra a probabilidade de falha referente a cada tipo de solo. 0 deslocamento relativo entre andares possui o valor mais alto no primeiro andar do edifício, conforme pode ser observado nas Tabs. 3,4 e 5 . Verifica-se que as simulações referentes aos solos moles e rígidos apresentam frequências médias de solo que estão localizadas entre o primeiro e segundo modos de vibrações da estrutura o que, consequentemente, induz na estrutura valores mais altos de amplitude de deslocamento e aceleração e acentua o deslocamento relativo entre os andares de modo que, essas simulações apresentam as maiores probabilidades de falha estrutural. As simulações referentes ao solo tipo rocha são as que causam menos dano estrutural, pois a frequência média do solo está entre o segundo e terceiro modos de vibrações da estrutura. Em adição, destaca-se, também, que o valor do PGA médio adotado é um valor alto e que a estrutura escolhida para a análise não possui qualquer tipo de controle de vibrações o que justifica as altas probabilidades de falha estrutural em todos os tipos de solo. 


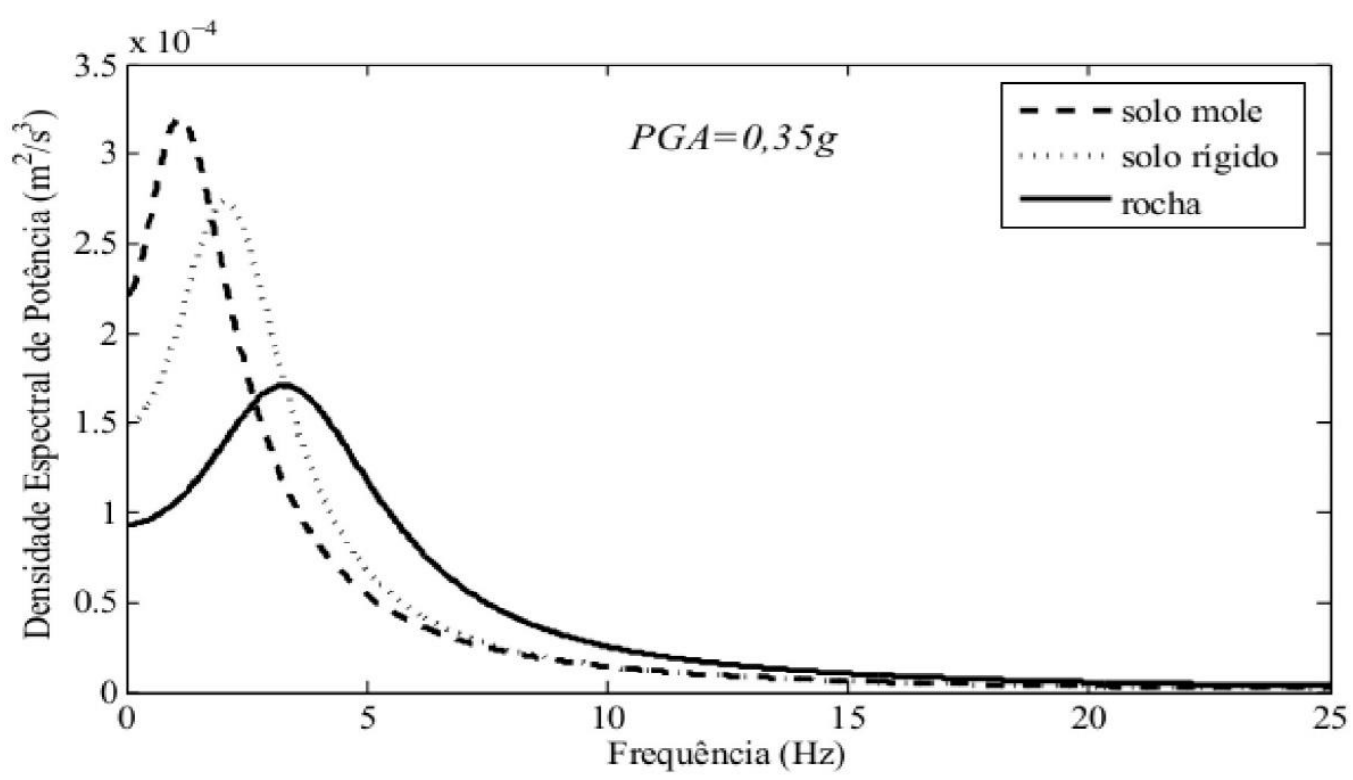

Figura 3: Espectros de potência.

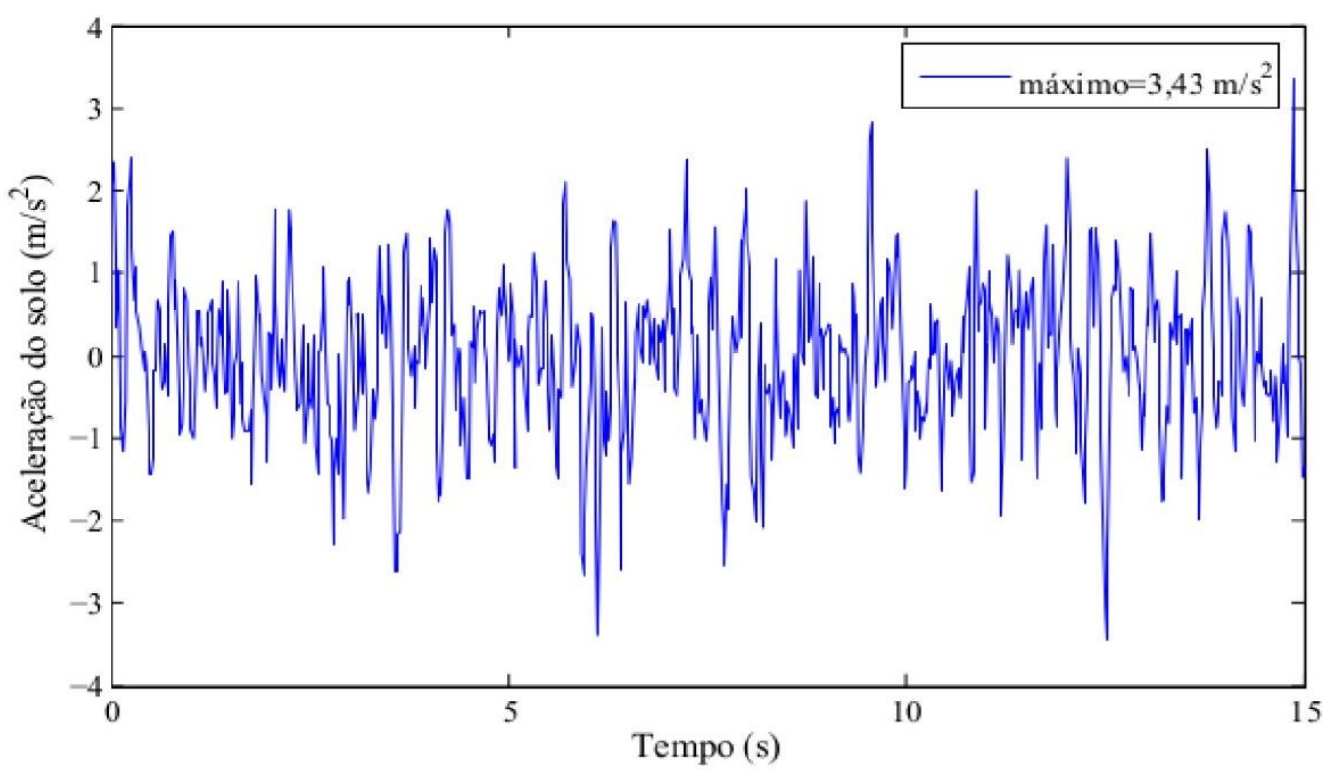

Figura 4: Sinal sísmico.

Tabela 3. Resultados médios do solo mole.

\begin{tabular}{crcc}
\hline Andar $(i)$ & $M\left(\max \left|z_{i}\right|\right)(m)$ & $M\left(\max \left|\ddot{z}_{i}\right|\right)\left(m / s^{2}\right)$ & $M\left(\max \left|\Delta_{i}\right|\right)(m)$ \\
\hline 1 & 0,0466 & 4,7071 & 0,0466 \\
2 & 0,0915 & 7,6704 & 0,0450 \\
3 & 0,1327 & 9,4751 & 0,0425 \\
4 & 0,1724 & 10,5780 & 0,0395 \\
5 & 0,2071 & 11,3210 & 0,0360 \\
6 & 0,2373 & 11,8310 & 0,0321 \\
7 & 0,2626 & 12,3600 & 0,0276 \\
8 & 0,2824 & 13,2600 & 0,0222 \\
9 & 0,2962 & 14,4660 & 0,0158 \\
10 & 0,3033 & 15,7250 & 0,0083
\end{tabular}


Tabela 4. Resultados médios do solo rígido.

\begin{tabular}{cccc}
\hline Andar $(i)$ & $M\left(\max \left|z_{i}\right|\right)(m)$ & $M\left(\max \left|\ddot{z}_{i}\right|\right)\left(\mathrm{m} / \mathrm{s}^{2}\right)$ & $M\left(\max \left|\Delta_{i}\right|\right)(m)$ \\
\hline 1 & 0,0365 & 4,9016 & 0,0365 \\
2 & 0,0713 & 7,9294 & 0,0349 \\
3 & 0,1034 & 9,5582 & 0,0326 \\
4 & 0,1324 & 10,2950 & 0,0301 \\
5 & 0,1580 & 10,5040 & 0,0279 \\
6 & 0,1803 & 10,3760 & 0,0256 \\
7 & 0,1993 & 10,3390 & 0,0227 \\
8 & 0,2147 & 11,2300 & 0,0189 \\
9 & 0,2258 & 12,7280 & 0,0138 \\
10 & 0,2316 & 14,2190 & 0,0074 \\
\hline
\end{tabular}

Tabela 5. Resultados médios do solo tipo rocha.

\begin{tabular}{crcc}
\hline Andar $(i)$ & $M\left(\max \left|z_{i}\right|\right)(m)$ & $M\left(\max \left|\ddot{z}_{i}\right|\right)\left(\mathrm{m} / \mathrm{s}^{2}\right)$ & $M\left(\max \left|\Delta_{i}\right|\right)(\mathrm{m})$ \\
\hline 1 & 0,0261 & 5,2178 & 0,0261 \\
2 & 0,0506 & 7,9045 & 0,0247 \\
3 & 0,0727 & 8,9344 & 0,0227 \\
4 & 0,0923 & 9,2025 & 0,0210 \\
5 & 0,1092 & 9,2244 & 0,0197 \\
6 & 0,1238 & 8,9222 & 0,0183 \\
7 & 0,1365 & 8,5739 & 0,0166 \\
8 & 0,1469 & 8,7906 & 0,0143 \\
9 & 0,1548 & 9,9560 & 0,0109 \\
10 & 0,1592 & 11,7130 & 0,0061 \\
\hline
\end{tabular}

Tabela 6. Probabilidade de falha conforme o tipo de solo.

\begin{tabular}{cc}
\hline Tipo de solo & Probabilidade de falha \\
\hline Rocha & $28,2 \%$ \\
Rígido & $69 \%$ \\
Mole & $89,5 \%$ \\
\hline
\end{tabular}

\section{Conclusão}

No presente trabalho, foram apresentados os resultados numéricos de um modelo probabilístico sísmico, com incertezas nos parâmetros do movimento do solo e da estrutura analisada. Desenvolveu-se uma rotina computacional, no programa MATLAB , que permitisse o cálculo da resposta dinâmica de um edifício, com $n$ graus de liberdade, sujeito a excitações sísmicas. Como exemplo, foram feitas algumas simulações considerando três tipos de solo e supondo que o edifício estivesse localizado em uma região com um valor significativo de PGA. Os valores máximos médios, em módulo, para o deslocamento e a aceleração da estrutura em cada andar, o deslocamento entre os andares e a probabilidade de falha foram calculados para cada tipo de solo.

Os resultados mostraram que as maiores amplitudes de deslocamento e aceleração, e os maiores valores para os deslocamentos relativos entre os andares e as maiores probabilidades de falha foram verificadas para os tipos de solo cuja frequência média do solo estava situada entre os dois primeiros modos de vibrações da estrutura, o que também foi agravado pelo fato de que o edifício não possui nenhum tipo de controle de vibrações. Sendo assim, os resultados obtidos estão em conformidade com os exemplos analisados. 
Agradecimentos: As autoras agradecem a CAPES e o CNPq pelo apoio financeiro.

\section{Referências}

1. Kanai, K. (1961). An empirical formula for the spectrum of strong earthquake motions. Bulletin Earthquake Research Institute, v. 39, p. 85-95.

2. Miguel, L.F.F., Miguel, L.F.F. and Lopez, R.H. (2016). Failure probability minimization of buildings through passive friction dampers. The Structural Design of Tall Special Buildings. doi:10.1002/tal.1287.

3. Miguel, L.F.F., Miguel, L.F.F. and Lopez, R.H. (2016). Simultaneous optimization of force and placement of friction dampers under seismic loading. Engineering Optimization, v. 48, p. 582-602.

4. Mohebbi, M., Shakeri, K., Ghanbarpour, Y. and Majzoub, H. (2013). Designing optimal multiple tuned mass dampers using genetic algorithms (gas) for mitigating the seismic response of structures. Journal de Vibration and Control, v. 19, p. 605-625.

5. PERU (2016). Decreto Supremo nro. 003-2016 - Vivienda.

6. Seya, H., Talbott, M.E. and Hwang, H.H.M. (1993). Probabilistic seismic analysis of a steel frame structure. Probabilistic Engineering Mechanics, v. 8, p.127-136.

7. Shinozuka, M. and Jan, C.M. (1972). Digital simulation of random processes and its applications. Journal of Sound and Vibration, v. 25, p. 111-128.

8. Singh, M.P. and Moreschi, L.M. (2001). Optimal seismic response control with dampers. Earthquake Engineering and Structural Dynamics, v. 30, p. 553-572.

9. Tajimi, H. (1960). A statistical method of determining the maximum response of a building structure during an earthquake. In Proceedings of 2nd World Conference in Earthquake Engineering. Tóquio. 